\section{Radiographic imaging following the use of absorbable internal fixation devices in orthopaedic surgery - the 'invisible' screws}

Ian C Duncan
FFRad(D)SA
Hans H Volkersz
FCS Orth (SA)
Sunninghill Medical Institute
Johannesburg

\section{Abstract}

We describe a single case report of the use of polylactic acid absorbable internal fixation screws (Biofix, Bioscience Ltd, Tampere, Finland) for the operative repair of a Pipkin type IV hip fracture. Five months later a diagnosis of secondary avascular necrosis of the same femoral head was made on magnetic resonance imaging with no image degradation due to the presence of the screws. The use of biodegradable internal fixation devices therefore has the added advantage of allowing artefact-free postoperative imaging. metallic devices can result in significant imaging artefacts particularly with magnetic resonance imaging, which can result in sub-optimal postoperative imaging.

The use of absorbable fixation devices allows the radiologist the opportunity of obtaining optimal artefact-free postoperative imaging as an additional advantage.

\section{Case report}

An adult male patient was admitted following a motor vehicle accident having sustained a Pipkin type IV fracture of the left femoral head and acetabulum (Fig. 1a). At surgery, the acetabular fragment was removed and the femoral head fragment was reduced and fixed with two absorbable polylactic acid (PLA) screws (Biofix, Bioscience Ltd, Tampere, Finland).

The postoperative control radiograph showed good reduction of the hip. The bioabsorbable screws were not visible radiographically (Fig. 1b).

Five months later the patient presented with progressive pain in the same hip. Plain films taken at this stage showed maintenance of reducthe more traditional metallic rods, wires and screws. The presence of

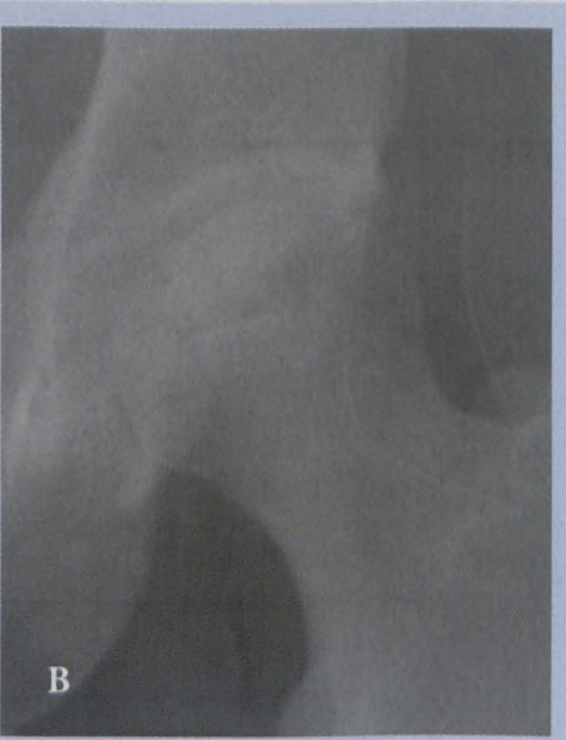

Figs. 1a and b. Pipkin type IV fracture before (a) and after (b) operative reduction and intemal fixation. The screw tracts are invisible. 


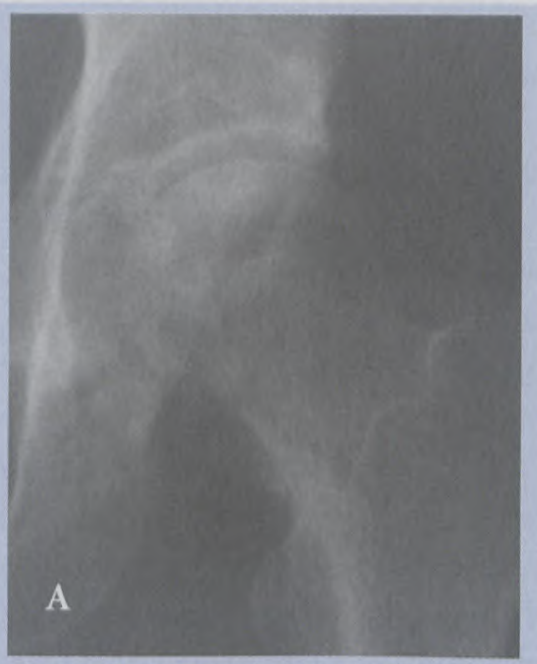

Fig. 2 a. Several months later plain film studies show healing of the fracture with normal alignment but no features of avascular necrosis.

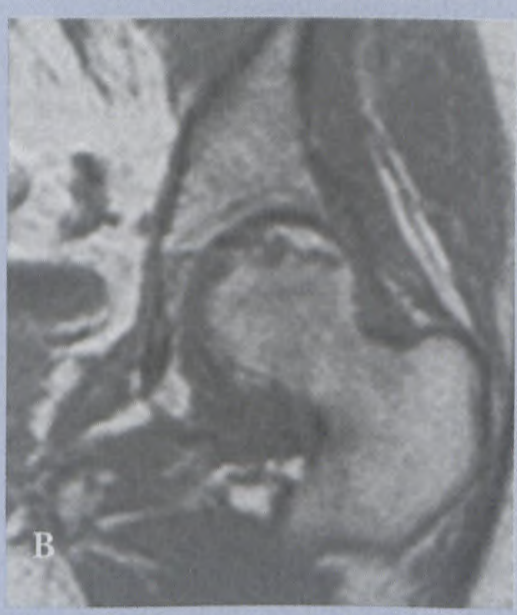

Fig. 2b. Coronal Tl-weighted MR image obtained 5 months later showing evidence of avascular necrosis in the superior aspect of the femoral head. There is no artifact due to the screws allowing clear visualisation of these changes.

tion and evidence of healing of the femoral head with no evidence of avascular necrosis (Fig. 2a). A magnetic resonance (MR) scan was obtained which showed no magnetic susceptibility artefact due to the presence of the polylactic acid screws (Fig. 2b).

\section{Discussion}

The first reported clinical use of absorbable internal fixation devices occurred in Finland in 1984 in a number of ankle fracture repairs. ${ }^{1}$ Since then, the use of these devices as well as the spectrum of available implants have increased considerably.

The materials used in the manufacture of these devices are synthetic polymeric composites, the most important of these being polyglycolic acid (PGA) and polylactic acid (PLA). In vivo these two substances are altered by hydrolysis to glycolic acid and lactic acid, both of which are naturally occurring metabolic products. These are then incorporated into various normal biochemical pathways including protein synthesis and energy production.

The shear strength values of these materials are 20 - 30 times those of cancellous bone, making them strong enough for orthopaedic fixation and weight-bearing. They demonstrate a flexibility part way between cancellous and cortical bone to allow micromovements essential for rapid bone healing. The speed of absorption of these devices is related to implant size and site, degree of metabolic activity and patient age. With absorption, the implants soften (PGA within 1 - 3 months and PLA within $3-12$ months) and total resorption occurs within $1-6$ years.

These devices include a number of fully-threaded and lag screws, rods, expansion plugs and anchors (Fig. 3). The rods are available in diameters of $1.1-4.5 \mathrm{~mm}$ and are used in the place of the more commonly implanted metallic rods and K-Wires. The devices are easily trimmed to an

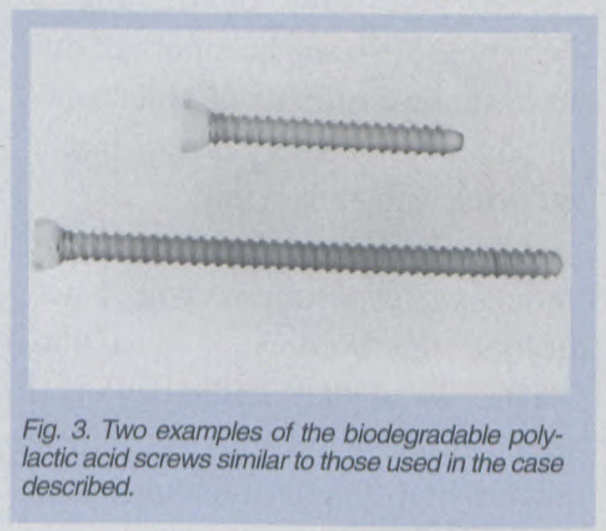

appropriate length. Indications for the use of these devices would include the repair of any fracture or other orthopaedic procedure where a wire, small rod, screw or tissue anchor would be used for internal fixation. ${ }^{2}$ There are even small devices available for the repair of certain meniscal tears in knee injuries. One major advantage of using these absorbable implants is the lack of any need for a secondary device removal procedure, resulting in reduced cost and risk of infection. ${ }^{3} \quad$ Unlike other metallic implants, the absorbable devices can be used in an infected area. These devices are especially useful for intraarticular fractures as a repeat surgery for removal of the screws or rods is not required.

Of interest to radiologists is that these devices are fully MRI compatible and do not degrade the MR image quality at all, ${ }^{4}$ allowing optimal postsurgical imaging where required. This adds yet another significant advantage to their usage. They are also completely non-opaque to X-ray imaging and thus cannot be seen on plain film or computed tomographic imaging, making them truly 'invisible' screws. A faint sclerotic margin to the screw tracts may sometimes be seen over time.

\section{References}

1. Rokkanen P, Bóstman $\mathrm{O}$, Vainionpää $\mathrm{S}$, et al. Biodegradable implants in fracture fixation: early results of treatment of fractures of the ankle. Lancet $1985 ; 22$ : 1422-1424.

2. Rokkanen $\mathrm{P}, \mathrm{Bóstman} \mathrm{O}$, Vanionpää $\mathrm{S}$, et al. Absorbable devices in the fixation of fractures. Trauma 1996; 40: 123-127.

3. Bóstman $\mathrm{O}$, Hirvensalo E, Partio E, Tormälä $\mathrm{P}$, Rokkanen P. Impact of the use of absorbable fracture fixation implants on consumption of hospital resources and economic costs. Trauma 1991; 31: 1400-1403.

4. Viljanen J, Kinnunen J, Bondestam S, Majola A, Rokkanen P, Törmälä P. Bone changes after experimental osteotomies fixed with absorbable self-reinforced Poly-L-lactide screws or metallic screws studied by plain radiographs, quantitative computed tomography and magnetic resonance imaging. Biomaterials 1995; 16: 13531358. 